\title{
Análise da Capacidade Funcional de Idosos Portadores de Diabetes Mellitus tipo 2
}

\section{Analysis of the Functional Capacity of Elderly People with Type 2 Diabetes Mellitus}

\author{
Maria de Fátima Alcântara Barros ${ }^{1}$ \\ Leônia Nayara Olegário Leite Souza e Silva² \\ Kássia Géssica Alves da Silva² \\ Savana Oliveira Henriques e Souza ${ }^{3}$ \\ Pamela Cristina Santos de Almeida ${ }^{3}$ \\ Maria das Graças Rodrigues de Araújo ${ }^{4}$ \\ Antonio Geraldo Cidrão de Carvalho ${ }^{1}$
}

\section{RESUMO}

Objetivo: Avaliar o equilíbrio, a marcha e a capacidade funcional em indivíduos com e sem neuropatia diabética. Metodologia: Realizouse um estudo transversal com usuários do SUS, residentes em João Pessoa. A amostra foi constituída por 44 usuários, selecionados por acessibilidade, com a idade variando de $60 \mathrm{a} \geq 71$ anos. Os participantes foram alocados em dois grupos: GC - diabéticos sem neuropatia $(n=22)$ e $G N$ - diabéticos com neuropatia $(n=22)$. A análise dos dados foi realizada por meio de estatística descritiva e inferencial. $O$ teste $t$ de Student foi empregado para verificar a igualdade entre as médias das variáveis. O Teste de Pearson foi utilizado para investigar a correlação entre as variáveis. O nível de significância utilizado foi de 5\%. Resultados: Em relação à escolaridade, $41,0 \%$ dos integrantes do GC e $55,0 \%$ dos participantes do GN concluíram o ensino fundamental $(p<0,001)$ $68,0 \%$ e $81,0 \%$ dos indivíduos, respectivamente, do GC e do GN possuíam uma renda per capita entre 1,3 a 2,5 salários mínimos $(p<0,001)$. A associação do diabetes com a hipertensão arterial esteve presente em ambos os grupos, acometendo $64,0 \%$ do GC e $73,0 \%$ do GN ( $p<0,001$ ). Pela Escala de Tinetti a média dos escores dos grupos foi 10,53 para o GC e 7,27 para o GN $(p<0,001)$. No GN identificou-se uma correlação com o início da marcha, o comprimento e a continuidade do passo e a base de apoio $(p<0,05)$. Utilizando-se a Escala de Berg verificou-se que a média dos escores dos participantes do $\operatorname{GC}(48,86)$ foi maior que a dos usuários do $\mathrm{GN}(40,05)$ ( $p<0,001)$, evidenciando um déficit de equilíbrio nos participantes do $\mathrm{GN}$, aumentando o risco de quedas. De acordo com a Escala de Lawton verificou-se uma diferença entre as médias dos grupos GC $(17,56)$ e GN $(16,22)$ ( $p<0,001$ ), evidenciando que os participantes de ambos os grupos apresentaram um comprometimento para desempenhar as atividades instrumentais da vida diária - AIVDs. Conclusão: As alterações mais relevantes foram encontradas nos integrantes do GN no que concerne ao equilíbrio, às AIVDs e à marcha, como também nos episódios de quedas.

DESCRITORES

Diabetes Mellitus. Fisioterapia. Equilíbrio e Marcha. Quedas. Atividades da Vida Diária.

\section{ABSTRACT}

Objective: To assess the balance, gait and functional capacity of daily living in elderly people with and without diabetic neuropathy. Methodology: A cross-sectional study was conducted with SUS users, residing in João Pessoa. The sample consisted of 44 users, selected for accessibility, with ages ranging from 60 to $\geq 71$ years. Participants were allocated into two groups: CG - diabetics without neuropathy $(n=22)$ and $G N$ - diabetics with neuropathy $(n=22)$. Data analysis was performed using descriptive and inferential statistics. Student's t-test was used to verify equality between the means of the variables. Pearson's test was used to investigate the correlation between variables. The level of significance used was $5 \%$. Results: Regarding education, $41.0 \%$ of the members of the CG and $55.0 \%$ of the participants in the GN completed elementary school $(p<0.001) .68 .0 \%$ and $81.0 \%$ of individuals, respectively, from CG and GN had a per capita income between 1.3 to 2.5 minimum wages ( $p$ p 0.001). The association of diabetes with arterial hypertension was present in both groups, affecting $64.0 \%$ of the CG and $73.0 \%$ of the GN ( $p<0.001)$. According to the Tinetti Scale, the mean scores of the groups were 10.53 for the CG and 7.27 for the GN $(p<0.001)$. In the GN, a correlation was identified with the beginning of the gait, the length and continuity of the step and the support base $(p<0.05)$. Using the Berg Scale it was found that the average of the scores of the participants in the CG (48.86) was higher than that of the users of the GN $(40.05)(p<0.001)$, showing a deficit of balance in the participants of the $\mathrm{GN}$, increasing the risk of falls. According to the Lawton Scale, there was a difference between the means of the CG (17.56) and GN (16.22) groups ( $p$ $<0.001$ ), showing that the participants in both groups showed a commitment to perform the tasks instrumental activities of daily living. Conclusion: The most relevant changes were found in the members of the GN regarding balance, activities of daily living and gait, as well as in episodes of falls.

\section{DESCRIPTORS}

Diabetes Mellitus. Physiotherapy. Balance and March. Falls. Activities of Daily Living.

${ }^{1}$ Professor(a) Titular do Departamento de Fisioterapia/Laboratório de Fisioterapia na Saúde Coletiva - LabFISC do Núcleo de Estudos e Pesquisas Epidemiológicas em Fisioterapia e Saúde - NEPEFIS do Centro de Ciências da Saúde - CCS da Universidade Federal da Paraíba - UFPB, João Pessoa, Paraíba, Brasil.

${ }_{2}^{2}$ Fisioterapeuta. Pesquisadora do Laboratório de Fisioterapia na Saúde Coletiva - LabFISC do Núcleo de Estudos e Pesquisas Epidemiológicas em Fisioterapia e Saúde - NEPEFIS/CCS/UFPB, João Pessoa, Paraíba, Brasil.

${ }^{3}$ Fisioterapeuta. Aluna do Programa de Pós-Graduação em Fisioterapia -PPGFIS/CCS/UFPB e pesquisadora do Laboratório de Fisioterapia na Saúde Coletiva - LabFISC do Núcleo de Estudos e Pesquisas Epidemiológicas em Fisioterapia e Saúde - NEPEFIS/CCS/UFPB, João Pessoa, PB - Brasil.

${ }^{4}$ Professora Associada do Departamento de Fisioterapia/Laboratório de Cinesioterapia e Recursos Terapêuticos Manuais (LACIRTEM) do Centro de Ciências da Saúde da Universidade Federal de Pernambuco, Recife, Pernambuco, Brasil. 
iabetes Mellitus (DM) é uma síndrome metabólica de origem múltipla, decorrente da falta de insulina e/ ou da incapacidade de a insulina exercer adequadamente seus efeitos ${ }^{1}$.

O DM é frequentemente encontrado em dois tipos principais: o DM tipo 1, quando ocorre uma ausência total na produção de insulina no organismo, resultando de uma ação autoimune sobre as células pancreáticas $\beta$; e o DM tipo 2, que ocorre devido a uma resistência maior a insulina, o que deixa sua produção deficitária. O DM tipo 1 é encontrado em cerca de $20 \%$ dos casos, sendo mais comum entre as crianças; já o DM tipo 2 é mais dominante atingindo cerca de $80 \%$ dos casos e apresentando grande relação com a obesidade ${ }^{2,3}$.

A International Diabetes Federation IDF $^{4}$ comenta que desde a sua primeira edição no ano de 2000, a prevalência estimada de diabetes (tipo 1 e tipo 2 combinadas, ambas diagnosticadas ou não) em pessoas entre 20 e 79 anos de idade cresceu de 151 milhões ( $4,6 \%$ da população global da época) para 463 milhões $(9,3 \%)$ atualmente. Sem medidas suficientes para o enfrentamento dessa epidemia, é possível prever que 578 milhões de pessoas no mundo $(10,2 \%$ da população mundial) terão diabetes até 2030 e, esse número sobe para 700 milhões (10,9\%), em 2045. Estimou-se que em 2016, 1,6 milhões de pessoas perderam a vida por consequências da doença ${ }^{5}$.

No Brasil, a IDF ${ }^{4}$ traz dados estimados do ano de 2019, em torno de 16,8 milhões de pessoas com diabetes, projetando esses dados para os anos de 2030 e 2045, respectivamente de 21,5 milhões e 26 milhões de pessoas com diabetes.

Dentre as complicações crônicas do DM a neuropatia diabética (ND) está entre as mais comuns, atingindo cerca de 20 a $50 \%$ dos casos. A ND atinge o sistema nervoso periférico sensitivo, motor e autonômico e seu acometimento geralmente ocorre de forma simétrica e distal, podendo surgir de forma silenciosa ou sintomática. Diabéticos afetados com a ND apresentam déficits de sensibilidade (tátil, proprioceptiva, vibratória e cinestésica) e diminuição da amplitude de movimento, o que gera uma instabilidade postural e um aumento no risco de quedas ${ }^{6,7}$.

O pé diabético é uma das consequências da ND, que em decorrência da diminuição da sensibilidade dolorosa, térmica, tátil e proprioceptiva dificultam a percepção do paciente a estímulos externos, levando a ferimentos e ulcerações ${ }^{8}$.

O aumento na expectativa de vida populacional teve como consequência a elevação do número de idosos na população, o que gerou uma maior incidência de doenças crônicas e incapacidades funcionais se caracterizando como um desafio para o sistema de saúde ${ }^{9}$. Acometendo cada vez mais um maior número de pessoas idosas, a incapacidade funcional pode ser descrita como a dificuldade na realização das tarefas do dia-a-dia, sendo estas consideradas como necessárias para uma vida independente. Para avaliar a capacidade funcional de um idoso são observadas as atividades de vida diária (AVDs), as atividades instrumentais de vida diária (AIVDs) e a mobilidade voluntária do idoso ${ }^{10}$.

O processo de envelhecimento 
ocorre de maneira diferente para cada indivíduo, alterando a percepção do controle postural e, em portadores do diabetes mellitus, essas mudanças são ainda maiores, sofrendo influência direta de fatores externos, como o estilo de vida e as condições socioeconômicas ${ }^{11}$.

Podendo ser descrita como um acontecimento que inclui eventos internos e externos, as quedas causam efeitos diretos e/ou indiretos para a saúde dos indivíduos. Os fatores internos incluem as alterações fisiológicas provocadas pelo envelhecimento, a presença de doenças, fraqueza muscula e alterações de marcha. Já os fatores externos são aqueles relacionados ao ambiente (incluindo iluminação, superfícies escorregadias, objetos no caminho, presença de degraus, ausências de barras de apoio, roupas e calçados inadequados), sendo responsáveis por aproximadamente 30 a $50 \%$ das quedas ${ }^{12,13}$.

As quedas em idosos são consideradas uma das principais causas de morbidade e mortalidade entre essa população, gerando uma maior dependência para as atividades de vida diária (AVD) e diminuindo assim, sua autonomia. Estudos realizados demonstram uma associação entre a perda de sensibilidade plantar e os déficits de equilíbrio, aumentando os riscos de quedas ${ }^{7}$.

A fim de melhor mensurar o risco de quedas por meio da avaliação do equilíbrio, da marcha e da funcionalidade de cada idoso alguns instrumentos são de grande importância e tem sido desenvolvido com essa finalidade ${ }^{14}$. A Escala de equilíbrio de Berg é amplamente utilizada para a avaliação de equilíbrio em idosos, apresentando ótima confiabilidade e sendo de fácil aplicação ${ }^{15}$. A Escala de Tinetti permite observar alterações durante as fases da marcha e do equilíbrio ${ }^{16}$. Para a avaliação das atividades instrumentais de vida diária (AIVD) utiliza-se a Escala de Lawton, que determina a independência ou a dependência do indivíduo durante a realização de determinadas atividades necessárias no cotidiano ${ }^{17}$.

A fisioterapia atua na prevenção e promoção da saúde, atuando diretamente nas necessidades de cada indivíduo promovendo uma melhoria da qualidade de vida dos idosos diabéticos, visto que proporciona um melhor controle glicêmico, reduz os níveis de colesterol, diminui a hipertensão arterial e, consequentemente, melhora a capacidade funcional e os episódios de quedas nessa população ${ }^{18}$.

Neste contexto, o objetivo deste estudo foi avaliar o equilíbrio, a marcha e a capacidade funcional em indivíduos com e sem neuropatia diabética.

\section{METODOLOGIA}

Realizou-se um estudo do tipo transversal com usuários do sistema único de saúde (SUS), assistidos pelo Programa de Diabetes do Centro de Assistência Integrada à Saúde - CAIS de Jaguaribe, localizado em João Pessoa, no período de janeiro/2018 a maio/2019.

De um total de 72 diabéticos avaliados, 44 usuários foram selecionados por acessibilidade para composição da amostra do estudo, tendo a faixa etária variado de 60 a 82 anos. Os participantes foram alocados 
em dois grupos: Grupo 1 - portadores de diabetes sem neuropatia (grupo controle, $n=$ 22) e Grupo 2 - portadores de diabetes com neuropatia $(n=22)$.

Adotou-se como critério de inclusão ser portador de diabetes mellitus, com idade $\geq 60$ anos. Utilizou-se como critério de exclusão: pacientes portadores de ferimentos nos pés, amputados nos membros inferiores, com deformidades severas nos pés e com patologias que apresentassem condições desfavoráveis e significativas ao equilíbrio e a marcha do indivíduo.

A coleta de dados foi iniciada por meio de uma avaliação fisioterapêutica, pela qual foram levantadas informações relacionadas com o diabetes, como o tipo, o tempo de diagnóstico, o tipo de tratamento realizado, a presença de hipertensão arterial associada ou de outras complicações. Por meio da inspeção estática analisou-se a presença de calos, queratoses, verrugas, rachaduras, micoses e flictenas, além do aspecto da pele, a pilificação, a umidade dos pés, tipos de dedos e unhas. $\mathrm{Na}$ inspeção dinâmica observouse o tipo de marcha, a distribuição do peso corporal e o apoio do pé durante a marcha. $\mathrm{Na}$ palpação foi observada a presença de edema, com ou sem cacifo, a temperatura do pé e a presença de dor em repouso.

A sensibilidade tátil, térmica, protetora, vibratória e dolorosa dos pés foi avaliada, respectivamente, por meio da passagem de um chumaço de algodão na região lateral do pé, com a aplicação de tubos, um contendo água quente e outro com água gelada, encostados alternadamente na região plantar dos pés, do monofilamento de Semmes-Weinstein 5.07 de $10 \mathrm{~g}$, testado em nove pontos na região plantar (primeiro, terceiro e quinto dígito plantar; primeira, terceira e quinta cabeça dos metatarsos plantares, lateral esquerda e direita do meio plantar e calcâneo) e um na região dorsal (entre o primeiro e o segundo dedo), de um diapasão de $128 \mathrm{~Hz}$, seguindo as recomendações do Conselho Internacional sobre o Pé Diabético, testado em três pontos de cada pé: maléolo interno, falange proximal do hálux e no dorso do pé. Considerou-se a sensibilidade comprometida quando houve falta de sensibilidade em um dos pontos, após três tentativas consecutivas e de um instrumento pontiagudo (palito fixo) aplicado no mesmo local e maneira do monofilamento. O portador de diabetes indicava, verbalmente, quais as sensibilidades estavam presentes ou não.

A aplicação do monofilamento foi realizada com o usuário em decúbito dorsal e com os olhos fechados, perpendicular à pele, até curvá-lo e, assim mantido, por dois segundos, para então verificar se houve ou não sensibilidade ao mesmo. A incapacidade de sentir o monofilamento de $10 \mathrm{~g}$ em quatro ou mais pontos foi considerada ausência de sensibilidade na região plantar. Os reflexos profundos dos membros inferiores, o patelar e o aquileu, foram avaliados utilizando-se um martelo neurológico de inox da marca Profissional stainiess. Os pulsos pedioso e tibial posterior foram avaliados pelo método palpatório e classificados em palpáveis e não palpáveis.

Os instrumentos utilizados para a coleta dos dados foram uma ficha de avaliação fisioterapêutica, um questionário estruturado, abordando aspectos sociodemográficos e as Escalas de Tinetti, Berg e Lawton, para 
avaliação, respectiva, da marcha, equilíbrio e atividades instrumentais de vida diária (AIVD).

Utilizando-se a Escala de Tinneti avaliou-se a marcha dos usuários, observandose as fases de apoio e de balanço de cada membro e suas possíveis alterações. A escala tem uma pontuação máxima de 12 pontos. Quanto mais baixa a for a pontuação obtida, maior será o risco de quedas ${ }^{16}$.

Pela Escala de Lawton avaliou-se o nível de independência dos participantes para a execução das atividades instrumentais da vida diária, tais como o uso de telefone, o hábito de viajar, a realização de compras, o preparo das refeições, a realização do trabalho doméstico, o uso dos medicamentos e o manuseio do dinheiro. Os itens são pontuados de 1 a 3 pontos, sendo a pontuação máxima igual a 21 pontos. Quanto menor a pontuação, maior o nível de dependência do indivíduo ${ }^{17}$.

Com a utilização da Escala de Equilíbrio de Berg avaliou-se o equilíbrio em 14 situações, representativas de atividades do dia a dia, tais como: ficar de pé, levantar-se, andar, inclinar-se à frente, transferir-se, virarse, dentre outras. A pontuação máxima a ser alcançada é de 56 pontos e cada item possui uma escala ordinal de cinco alternativas variando de 0 a 4 pontos, de acordo com o grau de dificuldade. Quanto menor for a pontuação atingida pelo indivíduo, maior será o risco de quedas ${ }^{15}$.Cada ponto a menos entre os escores 56 a 54 pontos representa um risco de quedas de 3 a $4 \%$; entre os escores 54 e 46 esse número cresce para 5 a $8 \%$ de chances; e abaixo de 36 pontos o risco de quedas é extremamente alto, podendo chegar a quase $100 \%$ de chances ${ }^{14}$.
Os dados obtidos foram codificados, digitados em dupla entrada e validados utilizando-se o programa Epi-Info, versão 16.0. A análise estatística foi realizada por meio de estatística descritiva, tendo sido obtida a média, o desvio padrão e a porcentagem para cada variável avaliada, utilizando-se a planilha Microsoft Excel.

O teste $t$ de Student foi utilizado para verificar a igualdade entre as médias das variáveis avaliadas. O Teste de correlação de Pearson foi empregado com o intuito de investigar se existiu influência entre as variáveis. O nível de significância utilizado foi de $5 \%(p<0,05)$.

Todas as pacientes assinaram um Termo de Consentimento Livre e Esclarecido, de acordo com o que determina a Resolução CNS/MS n. 466/2012.

O projeto de pesquisa foi submetido e aprovado pelo Comitê de Ética em Pesquisa do Hospital Universitário Lauro Wanderley da Universidade Federal da Paraíba.

\section{RESULTADOS}

ATabela 1 descreve a amostra de acordo com as características sociodemográficas. A maior parte dos participantes do Grupo sem neuropatia (GC) pertence à faixa etária entre 60 e 70 anos (90,8\%), enquanto no Grupo com neuropatia (GN) a maioria situava-se na faixa etária entre 60 a 65 anos (40,9\%). O sexo feminino foi predominante no estudo tanto no grupo sem neuropatia como no grupo com neuropatia, $64,0 \%$ e $59,0 \%$, respectivamente $(p<0,001)$. No que se refere ao grau de escolaridade, $41,0 \%$ dos integrantes do grupo sem neuropatia e $55,0 \%$ dos participantes 
Tabela 1. Características sociodemográficas dos usuários diabéticos

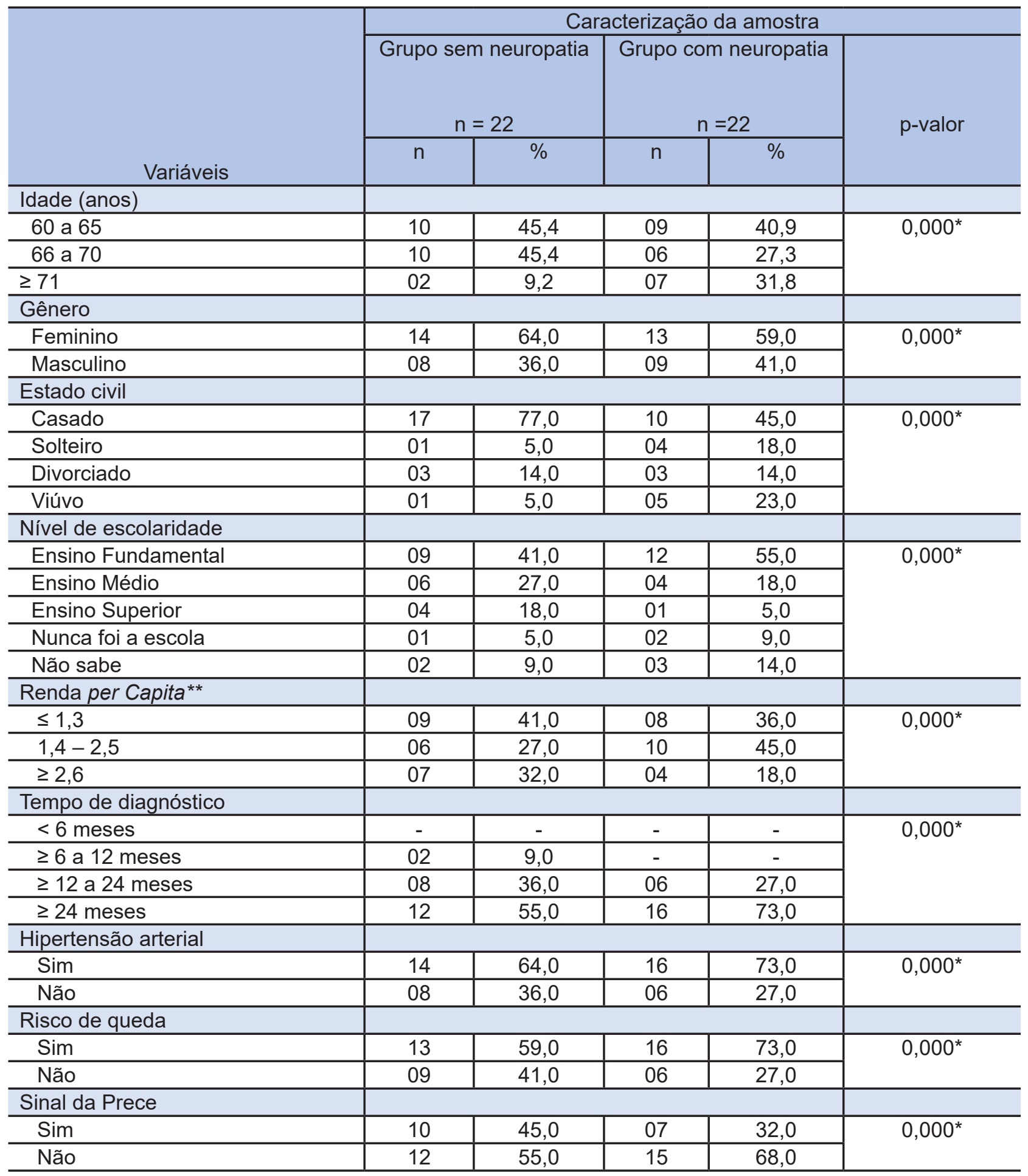

${ }^{*} p<0,001 .{ }^{* *}$ Salários mínimos. 
do grupo com neuropatia concluíram o ensino fundamental $(p<0,001)$. A respeito da renda, $68,0 \%$ e $81,0 \%$ dos indivíduos, respectivamente, do grupo sem neuropatia e do grupo com neuropatia possuíam uma renda per capita situada entre 1,3 a 2,5 salários mínimos ( $p<0,001)$. Em relação ao tempo de diagnóstico do diabetes, 55,0\% (grupo sem neuropatia) e 73,0\% (grupo com neuropatia) dos participantes apresentava 24 meses ou mais de diagnóstico $(p<0,001)$. A associação do diabetes com a hipertensão arterial esteve presente em ambos os grupos, acometendo $64,0 \%$ do grupo sem neuropatia e $73,0 \%$ do grupo com neuropatia $(p<0,001)$. Verificouse que $59,0 \%$ e $73,0 \%$ dos indivíduos, respectivamente, grupo sem neuropatia e grupo com neuropatia apresentaram risco de quedas ( $p<0,001$ ). Em 45,0\% (grupo sem neuropatia) e $32,0 \%$ (grupo com neuropatia) dos usuários o sinal da prece foi positivo $(p<$ 0,001 ), indicando uma redução da mobilidade articular nas extremidades dos membros superiores.
Conforme os dados da Tabela 2 foi possível perceber uma diferença na média dos escores dos grupos: 10,53 para o Grupo sem neuropatia e 7,27 para o Grupo com neuropatia $(p<0,001)$, indicativo de alterações na marcha. Verificou-se uma correlação entre não apresentar neuropatia com o comprimento $(p<0,05)$, a altura $(p<0,01)$ e a simetria do passo $(p<0,01)$, o desvio da linha reta $(p<$ $0,01)$ e o tronco $(p<0,01)$. No grupo com neuropatia identificou-se uma correlação com o início da marcha, o comprimento do passo, a continuidade do passo e a base de apoio $(p<$ $0,05)$. Entre os grupos com e sem neuropatia, todos os domínios foram significativos $(p<$ $0,001)$.

De acordo com os dados da Tabela 3 os grupos apresentaram uma diferença na média dos escores dos grupos: 48,86 do Grupo sem neuropatia e 40,05 do Grupo com neuropatia $(p<0,001)$, mostrando um déficit de equilíbrio presente nos participantes, principalmente no grupo com neuropatia, aumentando o risco de quedas. Observou-se ainda uma correlação

Tabela 2. Média e desvio padrão dos usuários, de acordo com a Escala de Tinetti

\begin{tabular}{l|c|c|c}
\hline \multirow{2}{*}{$\begin{array}{c}\text { Variáveis } \\
\text { (Total de escore) }\end{array}$} & \multicolumn{3}{c}{ Escala de TINETTI } \\
\cline { 2 - 4 } & $\begin{array}{c}\text { Grupo sem } \\
\text { neuropatia } \\
n=22\end{array}$ & $\begin{array}{c}\text { Grupo com } \\
\text { neuropatia } \\
n=22\end{array}$ & p-valor \\
\cline { 2 - 4 } & Média \pm DP & Média \pm DP & $0,000^{*}$ \\
\hline Início da marcha (1) & $0,91 \pm 0,29$ & $0,68 \pm 0,48^{\varepsilon}$ & $0,000^{*}$ \\
\hline Comprimento do passo (2) & $1,77 \pm 0,43^{£}$ & $1,36 \pm 0,49^{\varepsilon}$ & $0,000^{*}$ \\
\hline Altura do passo (2) & $1,86 \pm 0,35^{\beta}$ & $1,32 \pm 0,48$ & $0,000^{*}$ \\
\hline Simetria do passo (1) & $0,86 \pm 0,35^{\beta}$ & $0,41 \pm 0,50$ & $0,000^{*}$ \\
\hline Continuidade do passo (1) & $0,86 \pm 0,35$ & $0,50 \pm 0,51^{£}$ & $0,000^{*}$ \\
\hline Desvio da linha reta (2) & $1,68 \pm 0,48^{\beta}$ & $1,14 \pm 0,35$ & $0,000^{*}$ \\
\hline Tronco (2) & $1,77 \pm 0,43^{\beta}$ & $1,36 \pm 0,49$ & $0,000^{*}$ \\
\hline Base de apoio (1) & $0,82 \pm 0,39$ & $0,50 \pm 0,51^{£}$ & $0,000^{*}$ \\
\hline
\end{tabular}

${ }^{*} p<0,001$, teste t. Correlação de Pearson, ${ }^{\varepsilon} p<0,05$ e ${ }^{\beta} p<0,01$. 
Tabela 3. Média e desvio padrão dos usuários, de acordo com a Escala de Berg

\begin{tabular}{|c|c|c|c|}
\hline \multirow{3}{*}{$\begin{array}{c}\text { Variáveis } \\
\text { (Total de Escores) }\end{array}$} & \multicolumn{3}{|c|}{ Escala de BERG } \\
\hline & $\begin{array}{c}\text { Grupo sem } \\
\text { neuropatia } \\
n=22\end{array}$ & $\begin{array}{c}\text { Grupo com } \\
\text { neuropatia } \\
n=22\end{array}$ & $p$-valor \\
\hline & Média $\pm \mathrm{DP}$ & Média $\pm \mathrm{DP}$ & \\
\hline Transferências (12) & $9,64 \pm 1,57^{£}$ & $8,14 \pm 1,13$ & $0,000^{*}$ \\
\hline Provas estacionárias (16) & $15,32 \pm 1,29^{£}$ & $12,77 \pm 0,97$ & $0,000^{*}$ \\
\hline Alcance funcional (4) & $3,27 \pm 0,63$ & $3,05 \pm 0,38$ & $0,000^{*}$ \\
\hline Componentes rotacionais (12) & $10,86 \pm 1,42^{\beta}$ & $8,68 \pm 1,43$ & $0,000^{*}$ \\
\hline Base de sustentação (12) & $9,77 \pm 1,38^{\beta}$ & $7,41 \pm 1,14$ & $0,000^{*}$ \\
\hline Total de escores (56) & 48,86 & 40,05 & $0,000^{*}$ \\
\hline
\end{tabular}

${ }^{*} p<0,001$, teste t. Correlação de Pearson, ${ }^{\varepsilon} p<0,05$ e ${ }^{\beta} p<0,01$.

entre os dois grupos, senso significativa em relação às transferências, às provas estacionárias $(p<0,05)$, os componentes rotacionais e à base de sustentação $(p<0,01)$, no grupo sem neuropatia. Entre os grupos com e sem neuropatia, todos os domínios foram significativos $(p<0,001)$.

A partir dos dados da Tabela 4 observou-se uma pequena diferença na média dos escores dos grupos: 17,56 no Grupo sem neuropatia e 16,22 no Grupo com neuropatia
( $p<0,001$ ), evidenciando que os participantes de ambos os grupos de estudo apresentaram um comprometimento para desempenhar as atividades instrumentais da vida diária. Dentre os participantes do grupo com neuropatia, os indicadores que obtiveram menor média foram os referentes ao trabalho doméstico $(1,86 \pm 0,77)$, ao preparo de refeições $(2,14 \pm$ $0,83)$ e as compras $(2,27 \pm 0,77)(p<0,001)$. Identificou-se também uma forte correlação entre não apresentar neuropatia e o uso de

Tabela 4. Média e desvio padrão dos usuários, de acordo com a Escala de Lawton

\begin{tabular}{|c|c|c|c|}
\hline \multirow{3}{*}{$\begin{array}{c}\text { Variáveis } \\
\text { (Total de Escores = } 0 \text { a 3) }\end{array}$} & \multicolumn{3}{|c|}{ Escala de Lawton } \\
\hline & $\begin{array}{c}\text { Grupo sem } \\
\text { neuropatia } \\
n=22\end{array}$ & $\begin{array}{c}\text { Grupo com } \\
\text { neuropatia } \\
n=22\end{array}$ & p-valor \\
\hline & Média $\pm \mathrm{DP}$ & Média $\pm \mathrm{DP}$ & $0,000^{*}$ \\
\hline Uso de telefone & $2,32 \pm 0,78^{\beta}$ & $2,36 \pm 0,66$ & $0,000^{*}$ \\
\hline Relação à viagem & $2,68 \pm 0,48^{\beta}$ & $2,32 \pm 0,48^{£}$ & $0,000^{*}$ \\
\hline Relação às compras & $2,64 \pm 2,64^{\beta}$ & $2,27 \pm 0,77^{£}$ & $0,000^{*}$ \\
\hline Preparo de refeições & $2,50 \pm 0,86^{\beta}$ & $2,14 \pm 0,83$ & $0,000^{*}$ \\
\hline Trabalho doméstico & $2,14 \pm 0,83^{\beta}$ & $1,86 \pm 0,77$ & $0,000^{*}$ \\
\hline Uso de medicamento & $2,55 \pm 0,80$ & $2,50 \pm 0,51$ & $0,000^{*}$ \\
\hline Manuseio do dinheiro & $2,73 \pm 0,55^{\beta}$ & $2,77 \pm 043$ & $0,000^{*}$ \\
\hline Total de escores (21) & 17,56 & 16,22 & $0,000^{*}$ \\
\hline
\end{tabular}

${ }^{*} p<0,001$, teste t. Correlação de Pearson, ${ }^{⿷} p<0,05$ e ${ }^{\beta} p<0,01$. 
telefone, em relação à viagem e às compras, o preparo de refeições, o trabalho doméstico e o manuseio do dinheiro $(p<0,01)$. Em relação ao grupo com neuropatia existiu correlação, apenas, em relação à viagem e às compras $(p<0,05)$. Entre os grupos com e sem neuropatia, todos os domínios foram significativos $(p<0,001)$.

\section{DISCUSSÃO}

A partir dos dados encontrados na avaliação sociodemográfica dos usuários, observou-se que a maioria dos que fazem parte da faixa etária de 60 a 65 anos (40,9\%) apresentaram neuropatias decorrentes do diabetes. As características sociodemográficas mais evidentes no estudo são bem semelhantes com aquelas descritas por Tavares et al. ${ }^{19}$ que entrevistaram 113 idosos diabéticos em Uberaba (MG), com idade entre 60 e 80 anos, verificando que a maioria era do sexo feminino (72,6\%), casados ou em união estável $(50,5 \%)$ e com nível fundamental incompleto $(59,3 \%)$. Mendes et al. ${ }^{20}$ analisaram 872 idosos residentes na Cidade de São Paulo, tendo constatado que a prevalência do diabetes, em idosos, foi maior no sexo feminino $(18,89 \%)$ do que no sexo masculino (15,54\%). A faixa etária com maior prevalência foi de 70-79 anos $(20,61 \%)$ e a maioria com nível fundamental incompleto.

Outros estudos avaliando a prevalência de diabetes autorreferida também apontaram resultados parecidos quanto ao sexo, escolaridade e renda. Francisco et al. ${ }^{21}$ avaliaram 1.949 idosos no Estado de São Paulo, observando uma prevalência de 15,8\% nas mulheres e $14,9 \%$ nos homens. Menezes et al. ${ }^{22}$ fizeram a mesma análise, porém em idosos residentes em Campina Grande-PB. A amostra desse estudo foi composta por 806 idosos e a prevalência de diabetes referido foi de $14,7 \%$. Do total de idosos que referiram diabetes mellitus, $73,9 \%$ eram mulheres. O grupo etário mais prevalente foi de 60 a 69 anos com $48,7 \%$ dos casos. Prado at al. ${ }^{23}$ realizaram um estudo com 1.517 idosos de Campinas-SP, observando um maior percentual de diabéticos na faixa etária de 70 anos ou mais, com menor escolaridade e renda familiar per capita inferior a 1 salário mínimo. Por fim, Iser et al. ${ }^{24}$ fizeram seu estudo estimando a prevalência de diabetes autorreferido no Brasil no ano de 2013 , obtendo maior prevalência nas mulheres $(7,0 \%)$ comparativamente aos homens $(5,4 \%)$. A faixa etária com maior porcentagem de diabéticos foi de $65-74(19,9 \%)$, assim como os demais, o diagnóstico de diabetes foi referido com maior frequência por pessoas com ensino fundamental incompleto $(9,6 \%)$.

$\mathrm{O}$ diabetes mellitus e a hipertensão arterial fazem parte do grupo das doenças crônicas não transmissíveis (DCNT), que são a causa principal de mortalidade na maioria dos países de nosso continente, incluindo o Brasil, sendo responsáveis por cerca de $70 \%$ de todas as mortes no mundo ${ }^{25}$. São doenças com elevadas prevalências que se destacam entre os principais problemas de saúde pública na atualidade ${ }^{21}$, principalmente quando associadas, a hipertensão arterial e o diabetes mellitus, sendo relevantes causas de morbidade e mortalidade, com maior risco de doença renal, insuficiência cardíaca e acidente vascular encefálico ${ }^{21}$. Hu et al. ${ }^{27}$ comprovaram que a hipertensão e o diabetes tipo 2 aumentam o risco de acidente vascular encefálico de forma independente e a sua combinação aumenta drasticamente o risco.

Avaliando a presença concomitante 
neste estudo observou-se alta taxa de diabéticos que também eram portadores de hipertensão arterial (64\% do GC e $73 \%$ do GN). Os valores são semelhantes aos encontrados por Ferreira et al. ${ }^{28}$ que levantaram dados de usuários do serviço HiperDia, observando que a hipertensão arterial esteve presente em $80,9 \%$ dos pacientes com DM2, como também Prado et al. ${ }^{23}$ que detectaram que $74,8 \%$ dos idosos diabéticos entrevistados também sofriam de hipertensão arterial. Resultados semelhantes também foram encontrados em estudo realizado por Barros et al. ${ }^{29}$, onde foram identificados $66,6 \%$ de diabéticos em sua amostra.

Segundo o Relatório do Consenso da Associação Americana de Diabetes e da Sociedade Americana de Geriatria ${ }^{30}$, no diabetes a dor está sempre presente causando um déficit funcional e um risco elevado de quedas e fraturas.

Em relação ao risco de quedas neste estudo $73 \%$ dos usuários com neuropatia já tinham sofrido quedas constantes no seu cotidiano. Quando a queda ocorre em idosos torna-se necessário uma maior atenção, visto que ela é considerada um sinal de fragilidade e dependência. No estudo de Oliveira et al. ${ }^{31}$ encontraram uma taxa de quedas de $42 \%$ entre pacientes com diabetes mellitus tipo 2 quando comparados com pacientes sem diabetes $(33,8 \%)$.

Com base na escala de Tinetti, utilizada para avaliar o equilíbrio e as anormalidades da marcha, em usuários com e sem neuropatia, observou-se uma baixa pontuação nos escores totais, considerados normais. No grupo sem neuropatia foi significativamente marcante as alterações no comprimento, na altura e na simetria dos passos, como também o desvio da linha reta e do tronco. Em relação ao grupo com neuropatia as alterações foram mais evidenciadas no início da marcha, no comprimento e na continuidade dos passos, como também na base de apoio, ressaltando uma alteração importante no equilíbrio. Os resultados mostraram uma diminuição dos escores de ambos os grupos, sendo mais marcante no grupo com neuropatia, o qual apresentou maior risco de quedas. Saura et al. ${ }^{32}$ apontaram que a amplitude de movimento da articulação tíbio-társica está diminuída nos diabéticos, independente da presença ou ausência de neuropatia periférica, sendo este um dos fatores que dificulta a marcha e o equilíbrio, refletindo nos diabéticos com uma fase de apoio mais longa e a passos mais curtos $^{33}$. Cenci et al. ${ }^{6}$ descreveu que a perda da sensibilidade é um dos principais fatores que contribui para a diminuição de aferências para o sistema de controle motor e, portanto, para a diminuição do equilíbrio, ensejando que o grupo com neuropatia apresenta pontuação mais baixa em comparação com o grupo sem neuropatia.

Associado a esses fatores, temse na neuropatia diabética a perda da sensibilidade que gera alterações na marcha e na estabilidade corporal, passos mais curtos e com menor aceleração, assim como lentidão na correção de erros motores, além de comprometimento da sensação periférica, do tempo de reação e do equilíbrio ${ }^{6}$.

Os dados desse estudo corroboram com aqueles apresentados por Yavuzer et al. ${ }^{34}$, tendo como objetivo definir os desvios da marcha em pacientes com diabetes mellitus. $\mathrm{O}$ grupo com a neuropatia revelou marcha mais lenta, passos mais curtos, mobilidade limitada do joelho e tornozelo, menor momento flexor plantar do tornozelo e força do que o grupo diabético sem neuropatia e o controle, onde 
a diferença foi estatisticamente significativa.

De acordo com o estudo realizado por Ricci ${ }^{35}$, verificou-se um desempenho menor nos testes de equilíbrio dos portadores de neuropatia diabética, quando comparados aos resultados dos portadores de diabetes sem neuropatia. É reconhecido na literatura que a velocidade da marcha e o condicionamento físico estão diretamente ligados, onde um estilo de vida ativo melhora a marcha e estimula o equilíbrio.

Outro instrumento utilizado neste estudo foi a Escala de Equilíbrio de Berg, a qual mostrou, assim como a Escala de Tinetti, um déficit de equilíbrio, sendo mais evidente a diminuição dos escores no grupo com neuropatia.

A capacidade funcional pode ser descrita como a capacidade que o idoso apresenta de tomar decisões na sua vida de forma independente, enquanto a incapacidade funcional ocorre quando o idoso apresenta uma dificuldade ou precisa de ajuda para realizar alguma atividade do cotidiano ${ }^{36}$. De acordo com os resultados deste estudo, observou-se uma importante alteração nos indicadores relacionados com as atividades da vida diária. No grupo com neuropatia foi encontrado um resultado significativo em relação à viagem e a realizar compras, quando comparado com o grupo sem neuropatia, no qual foi identificada uma diminuição nos escores de todos os indicadores.

Com o avanço da idade, ocorrem mudanças fisiológicas como a diminuição da capacidade aeróbica, da força muscular, do equilíbrio e da flexibilidade, com alterações nas amplitudes de movimentos. Esses fatores repercutem sobre a capacidade funcional de realizar as atividades de vida diárias ${ }^{37}$.

\section{CONCLUSÃO}

Os resultados obtidos mostraram que os diabéticos portadores de neuropatia apresentaram alterações relevantes durante as fases da marcha, do equilíbrio e na execução de atividades da vida diária, apresentando uma maior probabilidade de sofrerem quedas, que podem causar complicações ainda mais graves.

Variáveis como a simetria do passo e o desvio ao andar em linha reta apresentaram média baixa, indicando que estes podem ser os domínios nos quais os diabéticos apresentam maior dificuldade na vida cotidiana. Em relação ao equilíbrio, o domínio base de sustentação apresentou uma média pouco expressiva, no grupo com neuropatia, evidenciando um maior risco de quedas. Nas atividades instrumentais da vida diária, o trabalho doméstico apareceu como sendo o domínio com maior dificuldade a ser realizado pelos portadores de neuropatia.

A partir dos resultados encontrados, ficou evidente a importância que deve ser dada pelos profissionais de saúde à prevenção e ao tratamento com o intuito de minimizar os sintomas e minimizar as complicações, tendo o fisioterapeuta um papel de grande importância nesse processo.

\section{REFERÊNCIAS}

1. Ministério da Saúde - Biblioteca Virtual em Saúde. Publicado 09 de Setembro de 2015.

2. Barbosa KGN. A complexa relação entre diabetes e doenças periodontais. Clipe Odonto - UNITAU. 2013; 5(1):65-71.

3. Sakae TM, Costa AWO, Linhares R. Prevalência dos fatores de

risco para Diabetes Mellitus tipo 1 no grupo de Endocrinologia Pediátrica do Hospital Universitário - UFSC. Arquivos Catarinenses de Medicina. 2004; 33(4): 23-30.

4. International Diabetes Federation. IDF Diabetes Atlas. 9. ed. Brussels, Belgium, 2019. Disponível em: https://diabetesatlas. org/en/. Acesso em: 16 de fevereiro de 2021. 
5. World Health Organization. Diabetes, 2020. Disponível em: www. who.int/news-room/fact-sheets/detail/diabetes. Acesso em: $16 \mathrm{de}$ fevereiro de 2021.

6. Cenci DR, Silva MD, Gomes EB, Pinheiro HA. Análise do equilíbrio em pacientes diabéticos por meio do sistema F-Scan e da Escala de Equilíbrio de Berg. Fisioter Mov. 2013; 26(1):55-61.

7. Pinheiro HA, Vilaça KHC, Carvalho GA. Estabilidade postural, risco de quedas e medo de cair com neuropatia diabética que realizam exercícios terapêuticos. Fisioter Pesq. 2014; 21(2): 127-32.

8. Ferreira LGF, Torre MV. Análise da correlação entre alterações sensitivas e mobilidade funcional em idosos diabéticos. Rev Fisioter S Fun. 2013; 2(1):42-49.

9. Silva RCP, Simões MJS, Leite AA. Fatores de risco para doenças cardiovasculares em idosos com diabetes mellitus tipo 2. Rev. Ciênc. Farm. Básica Apl. 2008; 28(1):113-121.

10. Franchi KMB, Monteiro LZ, Almeida SM, Pinheiro MHNP, Medeiros AIA, Montenegro RM, Júnior RMM. Capacidade funcional e atividade física de idosos com diabetes tipo 2. Revista Brasileira de Atividade Física \& Saúde. 2008; 13(3):158-66.

11. Fechine BRA, Trompieri N. O processo de envelhecimento: as principais alterações que acontecem com o idoso com o passar dos anos. Revista Científica Internacional 2012; edição 20, 1(7):106-132.

12. Jahana KO, Diogo MJD. Quedas em idosos: principais causas e consequências. São Paulo, Brasil, Editora Bolina, 2007. Rev Bras Fisioter. 2010; 14(6):491-496.

13. Oliveira AS, Trevizan PF, Bestetti MLT, Melo RC. Fatores ambientais e risco de quedas em idosos: revisão sistemática. Rev. Bras. Geriatr. Gerontol. 2014; 17(3):637-45.

14. Pimentel RM, Scheicher ME. Comparação do risco de queda em idosos sedentários e ativos por meio da escala de equilíbrio de Berg. Fisioter Pesq. 2009; 16(1):6-10.

15. Berg KO, Wood-Dauphinee SL, Williams JI, Gayton D. Measuring balance in the elderly: validation of an instrument. Can J Public Health. 1992; 83(suppl 2):S7-S11.

16. Tinetti ME, Baker DI, Garret PG, Gottschalk CM, Koch ML, Horwitz RI. Yale Ficsit: Risk factor abatement strategy for fall prevention. J Am Geriatr Soc. 1993; 41(3):315 320.

17. Lawton MP, Brody EM. Assessment of older people: selfmaintaning and instrumental activities of daily living. Gerontologist. 1969; 9:179-86.

18. Mendonça SS, Morais JS, Moura MCGG. Proposta de um protocolo de avaliação fisioterapêutica para os pés diabéticos. Fisioter Mov. 2011; 24(2):285-28

19. Tavares MST, Rodrigues FR, Silva CGC, Miranzi SS. Caracterização de idosos diabéticos atendidos na atenção secundária. Ciênc. Saúde Coletiva. 20007; 12(5):1341-1352.

20. Mendes TAB et al. Diabetes mellitus: fatores associados à prevalência em idosos, medidas e práticas de controle e uso dos serviços de saúde em São Paulo, Brasil. Cad. Saúde Pública.2011; 27(6): 1233-1243.

21. Francisco PMSB, Segri NJ, Borim, Borim FSA, Malta DC. Prevalência simultânea de hipertensão e diabetes em idosos brasileiros: desigualdades individuais e contextuais. Ciênc Saúde Coletiva. 2018; 23(11):3829-3840.

22. Menezes NM, Sousa NDS, Moreira AS, Dixis FP. Diabetes mellitus referido e fatores associados em idosos residentes em Campina Grande, Paraíba. Rev Bras Geriatr. Gerontol. 2014; 17(4):829-839.
23. Prado MAMB, Francisco PMSB, Barros MBA. Diabetes em idosos: uso de medicamentos e risco de interação medicamentosa. Ciênc Saúde Coletiva. 2016; 21(11):3447-3458.

24. Iser BPM, Stopa SR, Chueiri OS, Szwarcwald CL, Malta DC, Monteiro HOC, Duncan BB, Schmit MI. Prevalência de diabetes autorreferido no Brasil: resultados da Pesquisa Nacional de Saúde 2013. Epidemiol. Serv. Saúde. 2016; 24(2):305-314.

25. Malta DC, Berna RTI, Lima MG, Araújo SSC, Silva MMA, Freitas MIF, Barros BA. Doenças crônicas não transmissíveis e a utilização de serviços de saúde: análise da Pesquisa Nacional de Saúde no Brasil. Rev Saude Publica. 2017; 51(Supl 1):4

26. Organização Mundial da Saúde - OMS. Resumo: Relatório mundia de envelhecimento e saúde. Genebra: OMS, 2015.

27. HU G, Sarti C, Pekka J, Peltonen M, Qiao Q, Antikainen R Tuomilethto $\mathrm{J}$. The impact of history of hypertension and type 2 diabetes at baseline on the incidence of stroke and stroke mortality. Stroke. 2005; 36(12):2538-2543.

28. Ferreira RAF, Ferreira MG. Características epidemiológicas de pacientes diabéticos da rede pública de saúde - análise a partir do sistema HiperDia. Arq Bras Endocrinol Metab. 2009; 53(1):80-86

29. Barros JLM. Intervenção Fisioterapêutica na neuropatia diabética em membros inferiores. Revista Presciência. 2012; 5:145-155.

30. Kirkman S, Briscoe VJ, Clark N, Florez H, Haas LB, Halter JB, et al. Diabetes nos Idosos: Um Relatório de Consenso da Associação Americana de Diabetes e da Sociedade Americana de Geriatria. Rev Portuguesa de Diabetes. 2013; 8(3):116-139.

31. Oliveiral PP, Fachinll SM, Tozattilll J, Ferreira MC, Marinheiro LPF. Análise comparativa do risco de quedas entre pacientes com e sem diabetes mellitus tipo 2. Rev Ass Médica Bras. 2012; 58(2):234-239

32. Saura V, Santos ALG, Ortiz RT, Parisi MC, Fernandes TD, Nery M Fatores preditivos da marcha em pacientes diabéticos neuropático e não neuropáticos. Acta Ortopédica Bras. 2010; 18(3):148-151.

33. Petrofsky JS, Lee S, Cuneo ML. characteristics in patients with type 2 diabetes; improvement after administration of rosiglitazone. Med Sci Monit. 2005; 11(6):143-151

34. Yavuzer G, Yetkin I, Toruner FB, Koca N, Bolukbasi N. Gait deviations of patients with diabetes mellitus: looking beyond peripheral neuropathy. Eura Medicophys. 2006; 42(2):127-133.

35. Ricci NA, Gazzola JM, Coimbra IB. Sistemas sensoriais no equilíbrio corporal de idosos. Arq Bras Ciên Saúde 2009; 34(2):94100.

36. Barbosa BR, Almeida JM, Barbosa MR, Barbosa LARR. Avaliação da capacidade funcional dos idosos e fatores associados à incapacidade. Ciênc Saúde Coletiva. 2014; 19(8):3317-25.

37. Mota TA, Alves MB, Silva VA, Oliveira FA, Brito PMC, Silva RS Fatores associados à capacidade funcional de pessoas idosas com hipertensão e/ou diabetes mellitus. Esc Anna Nery. 2020 24(1): e20190089.

\section{CORRESPONDÊNCIA}

Maria de Fátima Alcântara Barros

Laboratório de Fisioterapia na Saúde Coletiva - LabFISC

do Núcleo de Estudos e Pesquisa Epidemiológicas

em Fisioterapia e Saúde - NEPEFIS

do Centro de Ciências da Saúde da Universidade Federal da Paraíba. João Pessoa, PB- Brasil. CEP: 58051-900

E-mail: fatimalcan@yahoo.com 